\title{
Contribution of Fimbrial and Afimbrial Adhesins of Xylella fastidiosa to Attachment to Surfaces and Virulence to Grape
}

\author{
Helene Feil, William S. Feil, and Steven E. Lindow
}

University of California, Department of Plant \& Microbial Biology, Berkeley 94720-3102.

Accepted for publication 27 September 2006.

\begin{abstract}
Feil, H. Feil, W. S., and Lindow, S. E. 2007. Contribution of fimbrial and afimbrial adhesins of Xylella fastidiosa to attachment to surfaces and virulence to grape. Phytopathology 97:318-324.

The role of fimbrial and afimbrial adhesins of Xylella fastidiosa in biofilm formation was assessed by visualization of cell aggregates of mutant strains after incubation on glass surfaces. FimA- or FimFfimbrial mutants adhered as solitary cells at a slightly lesser frequency to glass surfaces than the parental strain; however, cell aggregates were not formed, unlike the wild-type strain. Conversely, whereas the XadA- and HxfB- nonfimbrial mutants also exhibited a much lower frequency of adherence to glass surfaces than the wild-type strain, most of the cells retained on the surfaces were in cell aggregates of different sizes, much

FimF- mutants did not form cell aggregates on glass surfaces when incubated as individual strains, aggregates of a FimA- or FimF- mutant were observed when co-incubated with either a XadA- mutant or HxfBmutant, respectively. These results are consistent with a model in which the fimbrial adhesins FimA and FimF are involved preferentially in cellto-cell aggregate formation whereas the afimbrial adhesions XadA and $\mathrm{HxfB}$ preferentially contribute to initial cell binding to surfaces, whereupon further cell aggregation can occur. In each of five separate experiments, FimA, FimF, XadA, and HxfB mutants of $X$. fastidiosa all were less virulent to grape than the corresponding wild-type strain. Fimbrial and afimbrial mutants might produce a reduced biofilm within vessels of grape and, hence, be deficient in various cell-density-dependent traits required for movement through the plant and, thus, virulence.
\end{abstract} like that of the parental strain. Neither fimbrial or afimbrial mutants formed a mature biofilm on the sides of flasks of broth cultures, unlike the dense biofilm formed by the wild-type strain. Although FimA- and
Additional keywords: hemagglutinin, Pierce's disease, pili.
Xylella fastidiosa is an important plant pathogen of a variety of economically important crops such as grape, almond, coffee, and citrus, as well as of various ornamental and woody plant species (5). X. fastidiosa also can colonize many other plant species without causing symptoms (19). Symptoms of most diseases caused by $X$. fastidiosa mimic those of water stress. Reduced flow of xylem sap is thought to result from occlusion of the xylem vessels by bacterial cells and their associated extracellular polysaccharides (EPS), as well as gums and tyloses of plant origin, perhaps as a response to initial xylem blockage by bacteria (3). Although disease severity is highly correlated with the proportion of vessels that are blocked by $X$. fastidiosa (15), this pathogen most commonly is found associated with the walls of xylem vessels in cell aggregates that did not occlude vessels $(1,15)$. This observation led to the suggestion that $X$. fastidiosa exists primarily in a commensalistic relationship with plants, during which it exploits the dilute nutrients in the xylem sap by forming a biofilm on the xylem vessel walls. This assemblage of cells could modify its local environment within the biofilm to better acquire nutrients from the flow of xylem sap past the biofilm, such as by producing EPS, which has been shown to enhance nutrient retention (2). Given that $X$. fastidiosa is found in xylem vessels throughout the plant after inoculation into discrete locations suggests that it can move readily between xylem vessels. Because the pits that connect xylem vessels are considered to be natural barriers for movement of bacteria, it is presumed that $X$. fastidiosa can breach such barriers by the production of extracellular enzymes such as cellulases and polygalacturonases. Indeed, an

Corresponding author: S. E. Lindow; E-mail address: icelab @ socrates.berkeley.edu

DOI: 10.1094/PHYTO-97-3-0318

(C) 2007 The American Phytopathological Society
$X$. fastidiosa mutant unable to produce polygalacturonase is avirulent and unable to move within the plant (24). The biofilm environment would be expected to be essential for the function of such extracellular enzymes in pit maceration; by restricting the diffusion of the enzyme away from the site of production, its availability for pit degradation could be maximized.

Attachment is important in various stages of biofilm formation. The process of forming an aggregated structure on a surface is initiated by attachment of formerly planktonic cells to a surface followed by proliferation of the cells and their self-association to form an aggregate (2). Often, such cells produce EPS and other substances to stabilize the biofilm and contribute to its functions in nutrient acquisition and defense against environmental extremes and predators (18). In some systems, the subsequent release of planktonic cells from the biofilm to initiate a biofilm at new sites is also an important component of the biofilm lifestyle (21). It seems clear that both the mechanism of attachment and its efficiency could vary in different phases of the process of biofilm formation. In the context of $X$. fastidiosa, it would appear that cells would need to be sufficiently adherent to the surfaces of xylem vessels so that they would be retained after introduction into the vascular system of a plant. On the other hand, if cells were too strongly attached to each other or to the surface that they could not escape the biofilm to cross the pit membrane to another vessel or to move to a more distal site within a given vessel, then its ability to colonize the plant would be restricted. Thus, the attachment process would be expected to be a multicomponent one or one which could be modulated in response to varying conditions within the biofilm, or both.

Attachment to host tissues is essential for successful infection by many microbial pathogens (23). Those microbial surface proteins that contribute to this process are commonly known as adhesins. Based on their size and structure, gram-negative bacterial 
adhesins have been classified as two types: fimbrial and nonfimbrial (10). X. fastidiosa has both types of adhesins. Dispersal of this pathogen occurs through an insect vector, and it has been suggested that these molecules also might be involved in attachment to insect cells (11).

Fimbrial adhesions include pili of various types appearing as filaments radiating from the surface of the bacterium. Pili allow cells to adhere to surfaces, including other cells. For example, pili often have been described as joining bacterial cells prior to transfer of DNA from one cell to another (often referred to as sex pili). X. fastidiosa produces two types of pili. Numerous short pili (from 0.4 to $1.0 \mu \mathrm{m}$ in length) encoded by a fimbrial operon comprising genes fimA, ecdD, fim $C, D, E$, and $F$ are observed on $X$. fastidiosa (14). In particular, FimA, the major subunit, and FimF, the anchor protein, appear necessary for proper assembly of the fimbrial structure (4). In addition, fewer, but much longer (1.0 to $5.8 \mu \mathrm{m}$ ) type IV pili, apparently encoded by a collection of 26 pil genes, are observed in X. fastidiosa (14).

$X$. fastidiosa also possesses putative afimbrial adhesions which are surface proteins contributing to the binding of cells to surfaces. $X$. fastidiosa harbors a homolog of xadA (encoding Xanthomonas adhesin-like protein A), which shares similarity with several nonfimbrial adhesins of animal pathogenic bacteria. This outer membrane protein is differentially expressed in hosts and is required for maximal virulence and normal colony morphology of Xanthomonas oryzae pv. oryzae (25). Xylella fastidiosa also harbors several hemagglutinins such as one similar to HecA in Erwinia chrysanthemi. HecA was required for maximum attachment of E. chrysanthemi to plant surfaces, aggregation of cells on the plant, cell killing, and virulence of this organism to tobacco seedlings (22). X. fastidiosa has four homologs to hecA, including those encoding HxfA (PD2118) and HxfB (PD1792). These hemagglutins are the largest genes in the $X$. fastidiosa genome. Previous studies showed that HxfA and HxfB mutants of $X$. fastidiosa were hypervirulent to grape and that these adhesins mediated contact between $X$. fastidiosa cells, which resulted in biofilm formation in vitro and biofilm maturation within xylem vessels (7).

Given that the process of colonization of xylem vessels by $X$. fastidiosa is apparently a progressive one, in which cells must colonize a given vessel sequentially before moving to adjacent vessels, it is impossible to predict the role of adhesions in the infection process; although adhesion-deficient cells would be free to spread more easily through a plant, the processes used to move through the plant might be inefficient if cells were altered in their ability to form a biofilm. In the present study, we compared the relative role of several fimbrial and afimbrial adhesins of $X$. fastidiosa to various stages of cell attachment to surfaces and biofilm formation in vitro as well as to their individual contribution to virulence to grape.

\section{MATERIALS AND METHODS}

Bacterial strains and culture media. $X$. fastidiosa grape strains 'Temecula 1' (ATCC 700964) and 'STL' (ATCC 700963), isolated from diseased grapevines (Vitis vinifera) in Temecula and Napa, CA, respectively, were used in all experiments. Electrocompetent Escherichia coli DH10B (Invitrogen, Carlsbad, CA) cells were used for propagation of plasmids. X. fastdiosa strains were grown on Periwinkle wilt medium with gelrite (PWG) solid medium (8) as in earlier studies (4). Biofilm development was observed in PWG without gelrite (PW broth) or PW broth in which the bovine serum albumin was replaced by fructose (1.0 g/liter) and glycerol (7.5 g/liter) (XFF broth).

Mutagenesis. FimA- (PD0062) and FimF- (PD0058) mutants of the $X$. fastidiosa grape strain Temecula were described previously (4). FimA- and FimF mutants of $X$. fastidiosa strain STL were made using the same gene replacement procedures and gene constructs used to make the corresponding mutants in strain Temecula. XadA- (PD0731) and HxfB- (PD1792) mutants also were produced using this gene replacement methodology. The sequence of the $X$. fastidiosa grape strain 'Temecula1' was used to determine the positions of fimbrial and afimbrial adhesions. Descriptions and positions of insertional disruptions of each gene are given in Table 1. Plasmid constructs and primers used in making gene replacements are given in Table 2. The 1,189-bp fragment chosen to produce chromosomal disruption of xadA started at position 1,447 of the 2,990-bp xadA gene. The 1,136-bp fragment chosen to disrupt chromosomal $h \times f B$ started at position 5,612 of the 10,130-bp $h x f B$ gene. Mutants were confirmed by polymerase chain reaction (PCR), sequencing, and Southern blots according to the methods of Feil et al. (4).

Adhesion assays. Wild-type, FimA, FimF, XadA, and HxfB mutants cells were inoculated individually into PW broth from inoculum scraped from PWG plates. Cells were cultured until an absorbance of 1.0 at $600 \mathrm{~nm}$ was measured for each cell suspension. Cell suspensions $(500 \mu \mathrm{l})$ were applied to glass slides and incubated for $4 \mathrm{~h}$. The slides then were rinsed by gently dipping them five times in a beaker filled with sterile water, and the remaining attached cells were stained with 4',6-diamidino-2phenylindole (DAPI) and examined under a fluorescence microscope. Similarly, glass slides were incubated with these cultures with shaking for $12 \mathrm{~h}$. The slides then were recovered from the flasks, rinsed, stained with DAPI, and examined under a fluorescence microscope.

The attachment of mixtures of $X$. fastidiosa fimbrial and afimbrial adhesin mutants were assessed by methods similar to above. Cells of mutant strains were stained with either PKH67 green fluorescent dye (Sigma-Aldrich, St. Louis) (FimA- or FimF- mutants) or PKH26 red fluorescent dye (Sigma-Aldrich) (XadA- or HxfB- mutants). These dyes did not affect cell viability because stained cells grew as well as nonstained cells on plates. Equal numbers of cells of two strains stained with either PKH67 or PKH26 were mixed and placed on a glass slide and incubated in a moist chamber for $8 \mathrm{~h}$, rinsed, and examined under the confocal laser-scanning microscope equipped with a metadetector. The "Meta" detector allows for the detection of multiple fluorescent dyes using a computational process called "linear unmixing." The process records each dye separately and separates each spectrum from the mixed dye sample. This allows each dye to be distinguished.

Biofilm formation. Biofilm formation was assessed in cells of wild-type or mutant strains grown in flasks containing either PW broth or XFF broth. Flasks (1 liter) containing $200 \mathrm{ml}$ of culture medium were inoculated with the various strains to a final concentration of $10^{8}$ cells $/ \mathrm{ml}$ and placed on a shaker at $28 \mathrm{C}$ for $24 \mathrm{~h}$. Biofilm formation was evaluated by visual examination of the size of the ring deposited on the glass. Cell counts were determined by dilution plating.

TABLE 1. Genes and insertion positions within the Xylella fastidiosa 'Temecula1' genome

\begin{tabular}{|c|c|c|c|c|c|c|}
\hline Gene & Call no. & Start codon & Stop codon & Size (bp) & $\begin{array}{l}\text { Position of kanamycin insertion } \\
\quad \text { (insert location in gene) }\end{array}$ & Predicted gene function \\
\hline FimA & PD0062 & 83,200 & 82,649 & 552 & $82,837(363)$ & Fimbrial subunit precursor/FimA \\
\hline FimF & PD0058 & 77,444 & 76,383 & 1,062 & $76,937(507)$ & Fimbrial adhesin precursor/MrkD \\
\hline XadA & PD0731 & 896,422 & 899,412 & 2,990 & $898,468(2046)$ & Outer-membrane protein/XadA \\
\hline HxfB & PD1792 & $2,092,276$ & $2,082,146$ & 10,130 & $2,086,996(5280)$ & Hemagglutinin-like protein \\
\hline
\end{tabular}


Pathogenicity assays. Grapevines of 'Cabernet sauvignon' (moderately susceptible to Pierce's disease) (5) or of 'Pinot Noir' (a more susceptible cultivar) were grown from dormant cuttings provided by the Foundation Plant Material Service, University of California, Davis. Grapevines were grown in a greenhouse until vines were $\approx 1 \mathrm{~m}$ long and then needle inoculated $(5 \mu \mathrm{l})$ with suspensions of $10^{8}$ cells/ml with FimA-, FimF-, XadA-, or HxfB- mutants, or with wild-type $X$. fastidiosa Temecula1 or STL as appropriate in a leaf petiole, according to the method of Hopkins and Adlerz (9). Inoculations were performed five separate times, each with cells of either a low-passage wild-type strain or with the mutants (FimA-, FimF, XadA-, and HxfB-) to ensure that virulence was not diminished due to a high number of passages in the laboratory. The mutant strains were recreated before each experiment to ensure that the mutants represented distinct mutational events. The inoculated grapevines were grown in a greenhouse and visualized for symptoms weekly. For the third experiment, the number of symptomatic leaves on each vine was recorded weekly. For each experiment, 10 control plants were inoculated with buffer only as negative control. Infection of vines by $X$. fastidiosa was confirmed by reculturing of the pathogen from symptomatic vines by the method of Hill and Purcell (8). Two months after inoculation, three infected petioles from each vine inoculated with either mutant or wild-type cells of either $X$. fastidiosa Temecula1 or STL were tested for the number of bacterial cells per gram of petiole tissue by dilution plating of tissue macerates by the method of Hill and Purcell (8). Mutant strains of $X$. fastidiosa always were recovered on both PWG and PWG containing kanamycin at $30 \mu \mathrm{g} / \mathrm{ml}$ whereas the wild-type strain was recovered only on PWG from all symptomatic leaves.

\section{RESULTS}

FimA- and FimF- mutants of $X$. fastidiosa strain Temecula were described previously (4). To compare the contribution of FimA and FimF to attachment and virulence, we constructed FimA- and FimF- mutants in X. fastidiosa strain STL, which is somewhat more virulent to grape than strain Temecula using the same methods and cloned fimA and fimF genes as before (4). XadA- and HxfB- mutants of $X$. fastidiosa grape strains Temecula and STL were produced using a similar gene replacement method described previously for FimA and FimF. These mutants were characterized by PCR, sequencing, and Southern blots to ensure that gene replacement had occurred (data not shown). Plasmids that have been described for $X$. fastidiosa $(6,20)$ are not sufficiently stable without antibiotic selection to enable complementation of mutant phenotypes in planta; therefore, we recreated fimbrial and afimbrial mutants several times to ensure that their phenotypes were always the same and, thus, not due to secondary mutations that might have occurred during the process of gene replacement.

Biofilm formation. Biofilm formation in a liquid culture of bacteria integrates the several processes involved in attachment to a solid surface and the accumulation of cells into a mass on that surface; therefore, we investigated the formation of rings of cells that form on the sides of flasks as an initial assessment of any deficiencies in this process exhibited by fimbrial or afimbrial adhesion mutants of $X$. fastidios $a$. The mass of the ring formed by wild-type $X$. fastidiosa Temecula was approximately threefold higher when cells were grown in fructose-containing XFF broth compared with PW broth (data not shown). Neither FimA-, FimF-, or XadA- mutants exhibited a biofilm ring on flasks when grown in XFF broth, whereas the HxfB- mutant formed a slight biofilm ring within $24 \mathrm{~h}$ of incubation. By this time, the wild-type strain had produced a dense biofilm ring that was at least 10 -fold greater in mass than that formed by even the HxfB- mutant (data not shown). An inverse relationship was observed between the mass of the biofilm ring formed on the culture flask and the number of cells remaining in suspension in the culture medium. The number of planktonic cells suspended in cultures of FimA-, FimF-, XadA-, and HxfB- cells was greater than $10^{8}$ cells $/ \mathrm{ml}$ after $24 \mathrm{~h}$ of incubation, whereas fewer than $\approx 10^{7}$ cells $/ \mathrm{ml}$ of the wild-type strain remained in suspension at this incubation time. Under the culture conditions that we employed, we did not see evidence that $X$. fastidiosa formed cell-to-cell aggregates within broth cultures but, instead, aggregated to each other only after some cells had attached to a surface.

Adhesion of $X$. fastdiosa mutants to glass. Wild-type cells of $X$. fastidiosa became attached to glass either after $4 \mathrm{~h}$ of exposure to a static culture (Fig. 1) or after $12 \mathrm{~h}$ of exposure to a shaking culture (Fig. 2). Cells occurred both as single cells and as small and large cell aggregates on the glass surface. The size of bacterial aggregates tended to increase with time of exposure of the cell suspensions to the glass surface (compare Figs. 1 and 2). In contrast, cells of FimA- and FimF- mutants occurred almost exclusively as solitary cells on glass surfaces, irrespective of how

TABLE 2. Plasmids and primers used in the study

\begin{tabular}{|c|c|c|}
\hline Plasmids and primers & Relevant characteristics, primer sequence & Source or reference \\
\hline \multicolumn{3}{|l|}{ Plasmids } \\
\hline pUC18 & pMB1 derivative, rep (pMB1), bla $\left(\mathrm{Ap}^{\mathrm{R}}\right)$, lacZ & Norrander et al. 1983 (17) \\
\hline pFAK & pFA containing 965-bp fragment with kan gene & Feil et al. 2003 (4) \\
\hline pFA2 & pUC18 containing 1,520 -bp fimA/kan fragment of $\mathrm{pFAK}$ & Feil et al. 2003 (4) \\
\hline pSF & pUC18 containing 1,097-bp fimF fragment of 'STL' & Feil et al. 2003 (4) \\
\hline pFFK & pFF containing 965-bp fragment with kan gene & Feil et al. 2003 (4) \\
\hline pXA & pUC18 containing 1,056-bp xadA fragment from 'Temecula1' & This work \\
\hline pXAK & pXA containing 965-bp fragment with kan gene & This work \\
\hline pHX & pUC18 containing $1,136-\mathrm{bp} h x f B$ fragment from 'Temecula1' & This work \\
\hline pHXK & pHX containing 965-bp fragment with kan gene & This work \\
\hline \multicolumn{3}{|c|}{1} \\
\hline PUC1 & 5'-CTTTACACTTTATGCTTCCGG-3' & $\ldots$ \\
\hline PUC2 & 5'-GCAAGGCGATTAAGTTGG-3' & $\ldots$ \\
\hline KAN1 & 5'-CTAGCAATTGCTGTCTCTTATACACATCTCAACCA-3' & $\ldots$ \\
\hline KAN2 & 5'-TTGCCAATTGAACCAATTCTGATTAGAAAAACTCA-3' & $\ldots$ \\
\hline FimA1 & 5'-GTCTGAGCTCCAACATCAAGTCGACCAAGTG-3' & $\ldots$ \\
\hline FimA2 & 5'-TATGGAGCTCCGACTCGACTGATAGGAGGC-3' & $\ldots$ \\
\hline FimF1 & 5'-GTCTGAGCTCCAACTTTGGAAACCAGATGTCG-3' & $\ldots$ \\
\hline FimF2 & 5'-TATGGAGCTCACTGGTATTCAATCGTGAAGGTT-3' & $\ldots$ \\
\hline XadA1 & 5'-AGTCGAGCTCAGAGTGCGACCAACGGTATC-3' & $\ldots$ \\
\hline XadA2 & 5'-CATGGAGCTCACTCACTCCCTGGGTTACCA-3' & $\ldots$ \\
\hline HxfB1 & 5'-CTGAACATCACCACCACCAC-3' & $\ldots$ \\
\hline $\mathrm{HxfB} 2$ & 5'-TGAAGAGGCTGTTGTTGGG-3' & $\ldots$ \\
\hline
\end{tabular}


long they were exposed to the glass surface (Figs. 1 and 2). Furthermore, while FimA- and FimF- mutants occurred as solitary cells on the glass surfaces, fewer cells of these mutants were observed adhering to glass at a given sample time compared with the wild-type strain (Figs. 1 and 2). Likewise, fewer solitary cells of XadA- or HfxB- mutants adhered to glass surfaces at a given sample time compared with the parental strain (Figs. 1 and 2). However, although XadA- and HfxB- mutants exhibited a lower spatial density on glass slides (adhered to fewer sites), the cells that adhered were most likely to be found in either small or large cell aggregates on slides (Fig. 2). In fact, whereas the wild-type strain adhered nearly twice as frequently as a single cell to glass surfaces, in either large or small aggregates, XadA- and HxfBmutants were nearly twice as likely to form either large or small cell aggregates as to adhere as single cells (Fig. 2). If one assumes that cell-to-cell aggregates form after initial cell attachment to
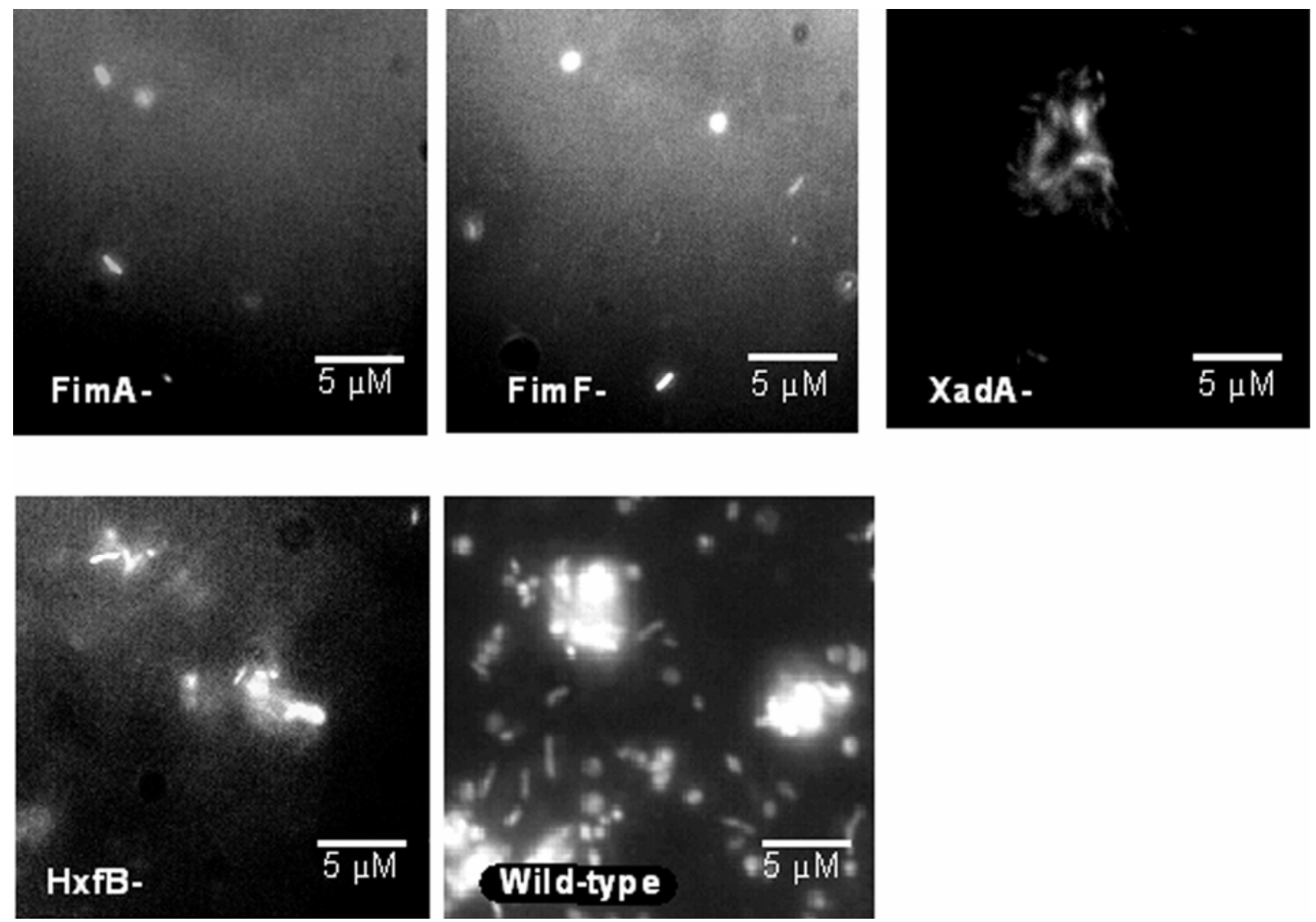

Fig. 1. 4',6-Diamidino-2-phenylindole-stained FimA-, FimF-, XadA-, HxfB, or wild-type Xylella fastidiosa cells that had attached to glass after $4 \mathrm{~h}$ incubation.

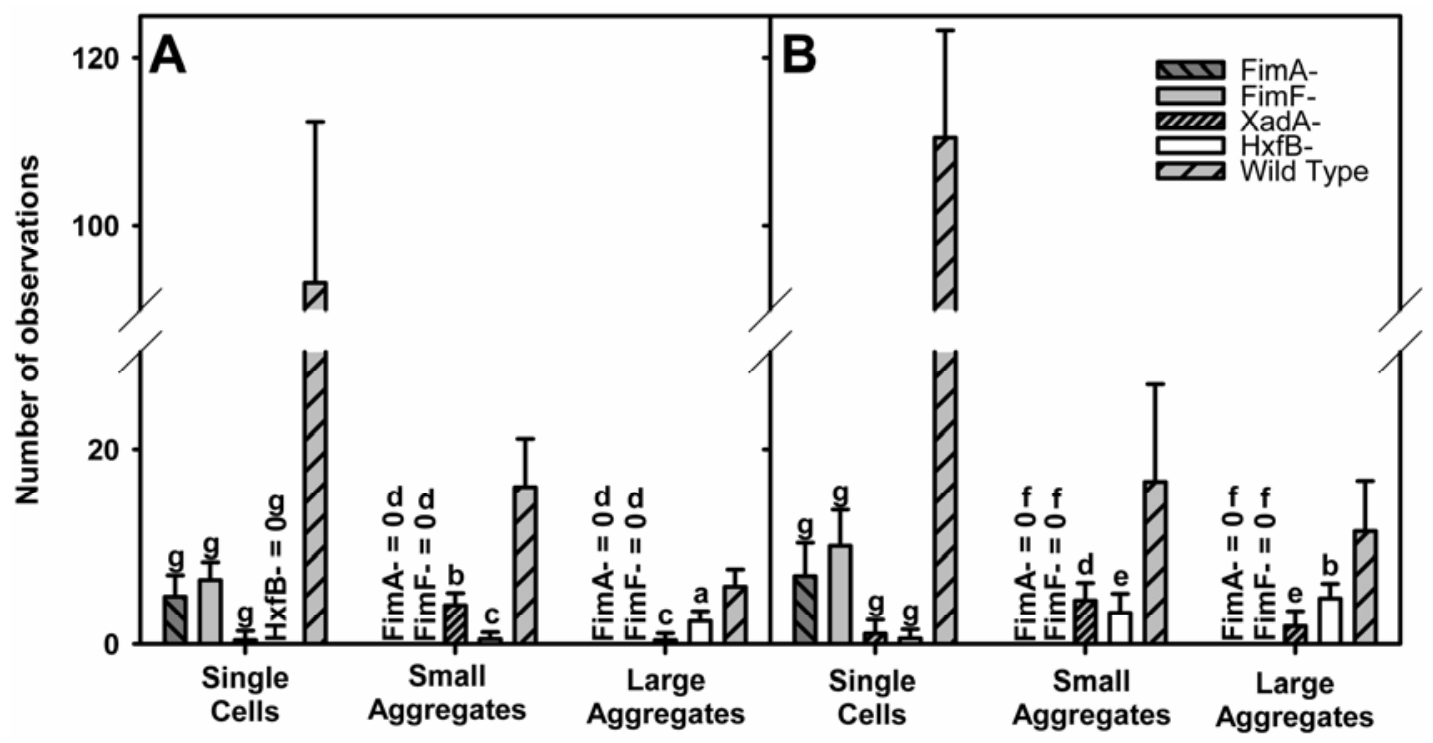

Fig. 2. Number of cells of wild-type Xylella fastidiosa or FimA-, FimF-, XadA-, and HxfB- mutants that had attached as single cells, small aggregates (i.e., <10 cells), or large aggregates ( $>10$ cells) to glass after $\mathbf{A}, 4$ or $\mathbf{B}, 12 \mathrm{~h}$ of incubation. Error bars indicate the standard deviation of the mean (number of samples $=10$ ), Letters above the bars indicates the level of significance with $P<10^{-3}(\mathrm{a}), 10^{-4}(\mathrm{~b}), 10^{-5}(\mathrm{c}), 10^{-6}(\mathrm{~d}), 10^{-7}(\mathrm{e}), 10^{-8}$ (f), and $10^{-10}$ (g) comparing mutant with wildtype for each aggregation category by a Student's $t$ test. 
surfaces, then it appears that FimA- and FimF- mutants as well as XadA- and HxfB- mutants all are less capable of initial attachment to glass surfaces compared with the wild-type strain. Given that XadA and HxfB- mutants were capable of forming cell aggregates on glass surfaces, unlike FimA- and FimF- mutants, it appears that FimA and FimF contribute to cell-to-cell adhesion that enables cell aggregates to form. Presumably, the higher number of solitary cells of FimA- and FimF- mutants compared with XadA- and HxfB- mutants that attached to glass reflects the greater contribution of XadA and $\mathrm{HxfB}$ to adhesion of $X$. fastidiosa cells to the glass surface. Thus, we speculate that the process of formation of cell aggregates on a glass surface involves a sequential contribution of primarily the afimbrial adhesions XadA and $\mathrm{HxfB}$ to initial attachment of cells to the abiotic surface; whereas, secondarily, cells are added to the initial surface colonists by a process involving pili that facilitates cell-to-cell attachment.

Attachment of mixtures of fimbrial and afimbrial adhesion mutants. To test the hypothesis that afimbrial adhesions are preferentially involved in initial attachment of $X$. fastidiosa cells to abiotic surfaces, we examined the spatial relationships of cell aggregates formed from mixtures of these mutants. Individual fimbrial mutants were treated with the green fluorescent strain PKH67 whereas afimbrial mutants were treated with the red fluorescent stain PKH26 and then mixed in equal numbers and allowed to attach to glass slides as above. These two fluorescent stains did not affect the viability of the bacterial mutants. Although FimA- and FimF- mutants did not form cell aggregates on glass surfaces when incubated as individual strains (Fig. 2), aggregates of FimF- (Fig. 3) mutant were observed when coincubated with either a XadA- mutant or HxfB- mutant. As observed with cells stained with DAPI, XadA- and HxfB- mutants preferentially formed cell aggregates on glass surfaces when stained with PKH26 before incubation (Fig. 3). Likewise, although solitary cells of FimF- mutants stained with PKH76 occasionally could be observed on glass slides, they were most often found together in aggregates with HxfB- mutants (Fig. 3). These results are consistent with the model that the fimbrial adhesions FimA and FimF are involved preferentially in cell-to-cell aggregate formation whereas the afimbrial adhesions XadA and HxfB preferentially contribute to initial cell binding to surfaces, whereupon further cell aggregation can occur.

Virulence of fimbrial and afimbrial adhesion mutants. In each of five separate experiments, FimA-, FimF-, XadA-, and HxfB- mutants of $X$. fastidios $a$ all were less virulent to grape than the corresponding wild-type strain (Figs. 4 and 5). These strains exhibited less disease at each sampling time after inoculation than the wild-type strain (Fig. 5). The disease incidence for plants inoculated with STL was higher than those inoculated with the Temecula strain for each genotype of cell, suggesting that STL is a more virulent strain of $X$. fastidiosa. No symptomatic leaves were recorded on the uninoculated control plants. The mutants were recreated a total of three times to test whether they exhibited similar virulence phenotypes; the mutants always conferred similarly low severity of disease in these replicate experiments, indicating that the disruption of the adhesin and not a mutation at another site was responsible for the reduced virulence. Although both fimbrial and afimbrial adhesin mutants all exhibited reduced virulence to grape, the temporal progress of disease was similar; disease symptoms appeared at similar times after inoculation.

\section{DISCUSSION}

The results of this study show that biofilm formation in $X$. fastidiosa is a complex process involving the contribution of both nonfimbrial and fimbrial adhesion factors. As measured by the ability to form dense biofilm rings in culture flasks, disruption of each fimbrial or afimbrial adhesin was sufficient to greatly reduce the formation of cell masses on this surface. This finding suggests that no single trait contributes preferentially to the formation of biofilms but, rather, it is most likely a sequential process involving several traits that each contribute to the assembly of cells adhering to both a solid surface and to each other. Because we did not see evidence that $X$. fastidiosa formed cell-to-cell aggregates except after some cells had attached to a surface, it appears that not only may several adhesins be required for aggregate formation, but that they might be differentially expressed during the process of biofilm formation. Thus, for example, if fimbrial adhesions are preferentially involved in cell-to-cell aggregation as suggested by the fact that FimA- and FimF- mutants did not form aggregates of any size on glass surfaces (Fig. 2), these genes might not be expressed in planktonic cells because such cells did not self-aggregate under the culture conditions used in this study. It is possible that fimA and fim $F$ are expressed only when local cell density increases, such as after the association of several cells on a surface. In such a setting, the diffusible signal factor (DSF), a molecule might increase locally in concentration (16), prompting the expression of genes whose expression is DSF dependent. Thus, fimA and fim $F$ might logically be expected to be expressed later in the process of biofilm formation and, hence, be under the control of the DSF signaling system. It also might be expected that the afimbrial adhesins such as XadA and HxfB might be expressed even in planktonic cells and, hence, enable adherence of such cells to surfaces immediately after their encounter without the need for changes in gene expression. Fimbrial adhesins appear more important in cell-to-cell aggregation than

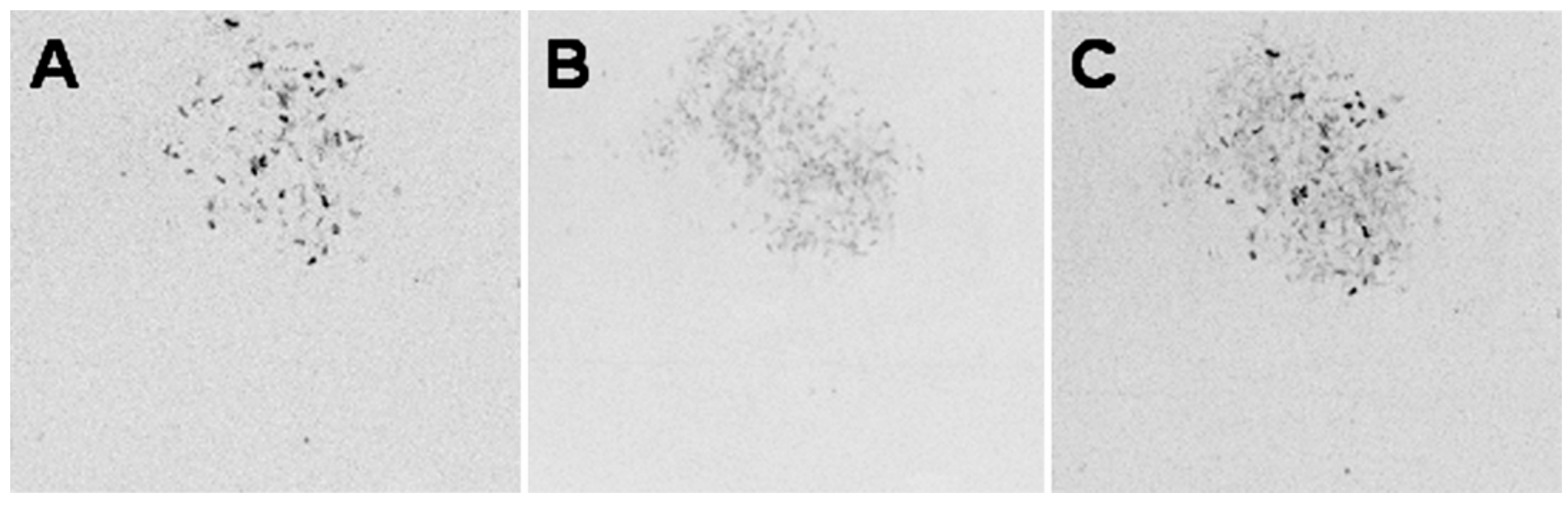

Fig. 3. Cells of an FimF- mutant strain of Xylella fastidiosa stained with PKH67 (A, depicted as dark cells) or of an HxfB- mutant stained with PKH26 (B, depicted as gray cells) after mixing in equal numbers and attachment to glass slides. Cells were viewed as $\mathbf{A}$ and $\mathbf{B}$, separated colors or $\mathbf{C}$, together using confocal microscopy equipped with a meta detector. 
the nonfimbrial adhesins because no cell aggregates were observed in FimA- or FimF- mutants (Fig. 2), and these mutants did not form a biofilm in flask cultures. This confirms the work of Meng et al. (14), who indicated that FimA mutants were biofilm deficient. Interestingly, biofilm formation as measured by ring formation on a glass flask was enhanced in XFF broth when compared with PW broth, indicating that fructose and glycerol may be inducers of biofilm formation or that bovine serum albumin inhibits biofilm formation. Similarly, Lemos et al. (13) and, later, Leite et al. (12) showed that biofilm and aggregate formation of $X$. fastidiosa was enhanced in glucose-based media more closely resembling xylem sap composition when compared with in the rich medium, PW broth.

Although both fimbrial and afimbrial adhesins appear to contribute to initial attachment to solid surfaces such as glass, XadA and HxfB appear to play a much more prominent role than FimA or FimF. Whereas only approximately half as many cells of FimA - or FimF- mutants adhered as single cells to glass surfaces, there were many more cells of these mutants found as single cells than of XadA- or HxfB- mutants (Fig. 2). These results suggest that the afimbrial adhesins played a dominant role in initial attachment to the surface. This finding is consistent with the observation that HecA, a homolog of $\mathrm{HxfB}$, was important in both attachment to and aggregation of cells of Erwinia chrysanthemi on Nicotiana clevelandii leaves (22). Our results are consistent with a model for biofilm formation of $X$. fastidiosa on surfaces that involves initial interaction of cells with surfaces via afimbrial adhesins followed by stabilization and addition of more cells to such initial colonists via pilus-mediated cell-cell interactions.

Although fimbrial and afimbrial adhesins both contribute to the virulence of $X$. fastidiosa to grape, no individual adhesin is required for pathogenicity. Plants inoculated with fimbrial or nonfimbrial mutants of $X$. fastidiosa all exhibited a lower incidence of disease (Figs. 4 and 5). In contrast to the results of Guilhabert and Kirkpatrick (7), the HxfB- mutants were always

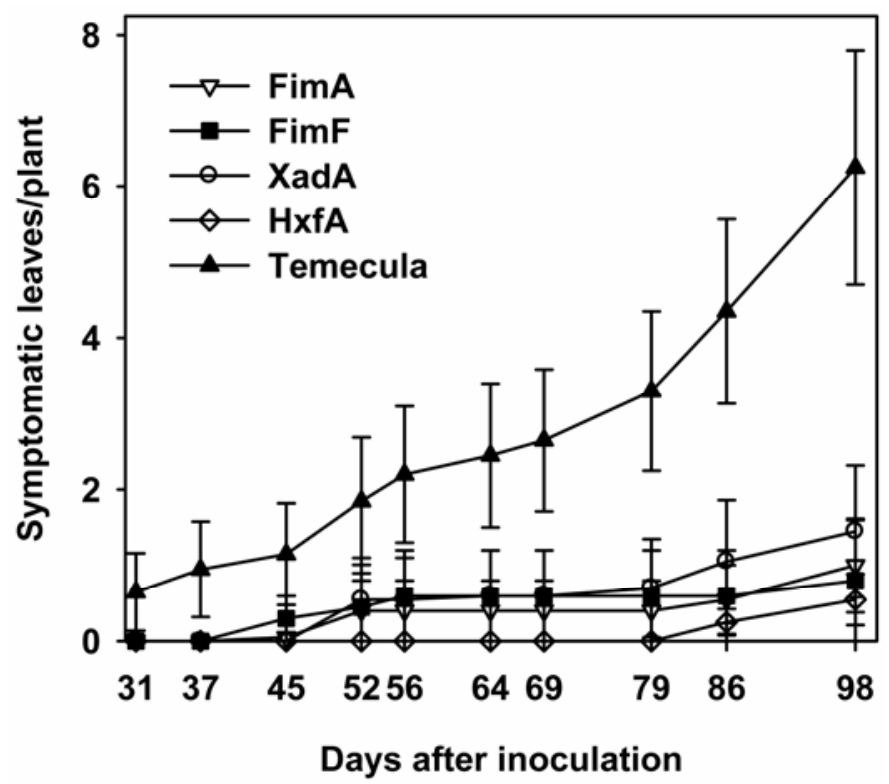

Fig. 5. Severity of Pierce's disease symptoms at various times after inoculation of Cabernet Sauvignon grape with FimA-, FimF-, XadA-, or HxfBmutants or the wild-type Xylella fastidiosa strain Temecula. Error bars indicate standard deviation of the mean (number of samples $=20$ ).
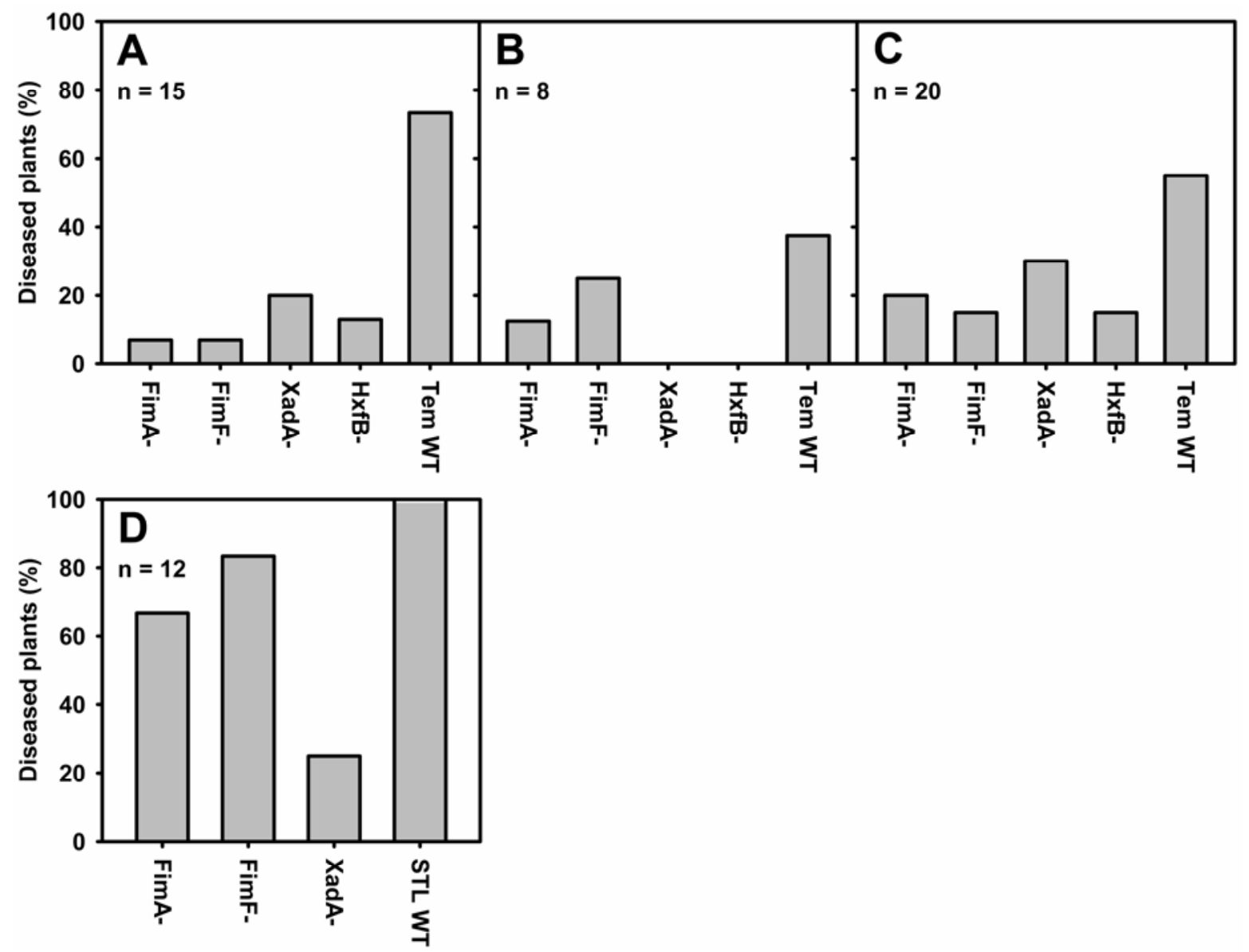

Fig. 4. Incidence of Pierce's disease to grape following inoculation with either FimA-, FimF-, XadA-, and HxfB- mutants or wild-type Xylella fastdiosa strain A, $\mathbf{B}$, and C, Temecula or D, STL in different experiments. A to D, A total of 15, 8, 20, and 12 plants, respectively, were inoculated with a given strain. 
less virulent to grape than the wild-type strain. The number of plants in which the HxfB- mutant was tested by Guilhabert and Kirkpatrick (7) was relatively small compared with this current study and it is possible that stochastic differences in symptom development might have obscured differences in virulence. For example, not all vines inoculated with wild-type cells exhibited symptoms, and disease severity was also variable between vines, necessitating the use of relatively large numbers of plants to distinguish among mutant strains. Alternatively, the greenhouse conditions under which disease was assessed might have differed, resulting in differential behavior of the mutants under these conditions. The HxfB mutant produced by Guilhabert and Kirkpatrick (7) had an insertion $721 \mathrm{bp}$ upstream from the HxfB insertion reported in this study (B. Kirkpatrick, personnel communication). However the orientation of the kanamycin insertion of the $h x f B$ gene in the Guilhabert and Kirkpatrick's study was not noted, so this may play a role in the way the downstream part of the gene was expressed and, consequently, it could have yielded different effects on virulence. In our study, the orientation of the kanamycin gene was opposite to the direction of transcription of the $h x f B$ gene to ensure that the kanamycin promoter would not read through. Perhaps this could account for the relatively large effect on virulence in our mutant (Figs. 4 and 5).

Although the process of multiplication and movement of $X$. fastidiosa in plants is not fully understood, we can speculate as to why fimbrial and afimbrial mutants that cannot form biofilms are less virulent to grape. The use of a green fluorescent proteinmarked strain of $X$. fastidiosa by Newman et al. (16) to examine the colonization of grape revealed that this pathogen was most frequently found in moderately sized cell aggregates that did not often block xylem vessels. A very high proportion of xylem vessels harbored at least some cells of $X$. fastidiosa, even in asymptomatic leaves. Symptom development in grape was strongly correlated with both the numbers of xylem vessels colonized by $X$. fastidiosa and the numbers of vessels blocked by cells of this pathogen (15). Thus, it appears that a major factor affecting virulence of this pathogen is its ability to move between xylem vessels from localized sites of inoculation. Furthermore, the movement of cells of $X$. fastidiosa from one xylem vessel to another was exclusively observed at sites of cell aggregates of intermediate size (15). Given that movement of $X$. fastidiosa may be a cell-density-dependent trait, and the observation that its virulence is strongly regulated via its production of the extracellular signaling molecule DSF, it appears that alterations of its ability to form biofilms within plants would reduce disease. By this model, fimbrial and afimbrial mutants would produce a reduced biofilm within vessels and, therefore, might not be expected to retain extracellular molecules such as pectinases required for movement and, hence, virulence (24. Some cell aggregates of fimbrial and afimbrial mutants still might form despite a lesser ability to form a mature biofilm or stochastically due to accumulation of planktonic cells at the ends of xylem vessels and so on; therefore, these mutants would be expected to retain the ability to cause disease, but with reduced virulence. Our understanding of the process of movement and of disease initiation will benefit from further understanding of cell-to-cell communication via DSF as well as an assessment of the aggregation status of fimbrial and afimbrial mutants within plants.

\section{LITERATURE CITED}

1. Alves, E., Marucci, C. R., Lopes, J. R. S., and Leite, B. 2004. Leaf symptoms on plum, coffee, and citrus and the relationship with the extent of xylem vessels colonized by Xylella fastidiosa. J. Phytopathol. 152:291-297.

2. Costerton, J. W., Lewandowski, Z., Caldwell, D. E., Korber, D. R., and Lappin-Scott, H. M. 1995. Microbial biofilms. Annu. Rev. Microbiol. 49:711-745.
3. Davis, M. J., Whitcomb, R. F., and Gillapsie, A. G., Jr. 1981. Fastidious bacteria of plant vascular tissue and invertebrates. Pages 2172-2188 in: The Prokarotes. M. P., Starr, H. Stolp, H. G. Truper, A., Balows, and H. G. Schlegel, eds. Springer-Verlag, Heilderberg, Germany.

4. Feil, H., Feil, W. S., Detter, J. C., Purcell, A. H., and Lindow, S. E. 2003. Site-directed disruption of the fimA and fimF fimbrial genes of Xylella fastidiosa Phytopathology 93:675-682.

5. Goodwin, P., and Purcell, A. H. 1992. Pierce's disease. Pages 76-84 in: Grape Pest Management, 2nd ed. R. C. Pearson and A. C. Goheen, eds. Division of Agriculture and Natural Resources, University of California, Oakland.

6. Guilhabert, M. R., and Kirkpatrick, B. C. 2003. Transformation of Xylella fastidiosa with broad host range RSF1010 plasmids. Mol. Plant Pathol. 4:279-285.

7. Guilhabert, M. R., and Kirpatrick, B. C. 2005. Identification of Xylella fastidiosa antivirulence genes: hemagglutinin adhesins contribute to $X$. fastidiosa biofilm maturation and colonization and attenuate virulence. Mol. Plant-Microbe Interact. 18:856-868.

8. Hill, B. L., and Purcell, A. H. 1995. Multiplication and movement of Xylella fastidiosa within grape and four other plants. Phytopathology 85:1368-1372.

9. Hopkins, D. L. 1989. Xylella fastidiosa: Xylem-limited bacterial pathogen of plants. Annu. Rev. Phytopathol. 27:271-290.

10. Hultgren, S. J., Abraham, S., Caparon, M., Falk, P., St Geme, J. W., III, and Normark, S. 1993. Pilus and nonpilus bacterial adhesins: assembly and function in cell recognition. Cell 73:887-901.

11. Lambais, M. R., Goldman, M. H. S., Camargo, L. E. A., and Goldman, G. H.. 2000. A genomic approach to the understanding of Xylella fastidiosa pathogenicity. Curr. Opin. Microbiol. 3:459-462.

12. Leite, B., Andersen, P. C., and Ishida, M. L. 2004. Colony aggregation and biofilm formation in xylem chemistry-based media for Xylella fastidiosa. FEMS Microbiol. Lett. 230:283-290.

13. Lemos, E. G., Alves, L. M., and Campanharo, J. C. 2003. Genomicsbased design of defined growth media for the plant pathogen Xylella fastidiosa. FEMS Microbiol. Lett. 219:39-45.

14. Meng, Y., Li, Y., Galvani, C. D., Hao, G., Turner, J. N., Burr, T. J., and Hoch, H. C. 2005. Upstream migration of Xylella fastidiosa via pilusdriven twitching motility. J. Bacteriol. 187:5560-5567.

15. Newman, K. L., Almeida, R. P. P., Purcell, A. H., and Lindow, S. E. 2003. Use of a green fluorescent strain for analysis of Xylella fastidiosa colonization of Vitis vinifera. Appl. Environ. Microbiol. 69:73197327.

16. Newman, K. L., Almeida, R. P. P., Purcell, A. H., and Lindow, S. E. 2004. Cell-cell signaling controls Xylella fastidiosa interaction with both insects and plants Proc. Natl. Acad. Sci. USA 101:1737-1742.

17. Norrander, J., Kempe, T., and Messing, J. 1983. Construction of improved M13 vectors using oligodeoxynucleotide-directed mutagenesis. Gene 26:101-106.

18. O’Toole, G., Kaplan, H. B., and Kolter, R. 2000. Biofilm formation as microbial development. Annu. Rev. Microbiol. 54:49-79.

19. Purcell, A. H., and Hopkins, D. L. 1996. Fastidious xylem-limited bacterial plant pathogens. Annu. Rev. Phytopathol. 34:131-151.

20. Qin, X., and Hartung, J. S. 2001. Construction of a shuttle vector and transformation of Xylella fastidiosa with plasmid DNA. Curr. Microbiol. 43:158-162.

21. Ramey, B. E., Koutsoudis, M, von Bodman, S. B., and Fuqua, C. 2004. Biofilm formation in plant-microbe associations. Curr. Opin. Microbiol. 7:602-609.

22. Rojas, C. M., Ham, J. H., Deng, W.-L., Doyle, J. J., and Collmer, A. 2002. HecA, a member of a class of adhesins produced by diverse pathogenic bacteria, contributes to the attachment, aggregation, epidermal cell killing, and virulence phenotypes of Erwinia chrysanthemi EC16 on Nicotiana clevelandii seedlings. PNAS 99:13142-13147.

23. Romantschuk, M., Roine, E., Ojanen, T., van Doorn, J., Louhelainen, J., Nurmiaha-Lassila, E.-J., and Haahtela, K. 1994. Fimbriae (pilus) mediated attachment of Pseudomonas syringae, Erwinia rhapontici, and Xanthomonas campestris to plant surfaces. Pages 67-77 in: Molecular Mechanisms of Bacterial Virulence. C. I. Kado and J. H. Crosa, eds. Kluwer Academic Publishers, Dordrecht, The Netherlands.

24. Roper, M. C., Greve, L. C., Labovitch, J. M., and Kirkpatrick, B. C. 2005. Polygalacturonase is required for Xylella fastidiosa colonization and pathogenicity in Vitis vinifera cv. Chardonnay grapevines. (Abstr.) Phytopathology 95(suppl.):S90.

25. Suvendra, K., Rajeshwari, R., Sharma, Y., and Sonti, R. V. 2002. A highmolecular-weight outer membrane protein of Xanthomonas oryzae pv. oryzae exhibits similarity to non-fimbrial adhesins of animal pathogenic bacteria and is required for optimum virulence. Mol. Microbiol. 46:637647. 